\title{
CORRECTION
}

\section{Correction to: Safety and Efficacy of Ripasudil in Japanese Patients with Glaucoma or Ocular Hypertension: 3-month Interim Analysis of ROCK-J, a Post-Marketing Surveillance Study}

Hidenobu Tanihara - Takahiko Kakuda - Tetsuro Sano •

Takashi Kanno · Ryosuke Imada · Wataru Shingaki · Ryoji Gunji

Published online: March 13, 2019

(c) Springer Healthcare Ltd., part of Springer Nature 2019

Correction to: Adv Ther (2019) 36:333-343

https://doi.org/10.1007/s12325-018-0863-1

In the original publication values in the results section are incorrect. Errors were also identified in Fig. 5. Both the updated results sections and Fig. 5 are provided below.

\section{RESULTS}

\section{Treatment Initiation Patterns with Ripasudil}

In the 3016 eyes (patients) included in the efficacy analysis, the numbers of patients classified as "Add-on (only)", "Add-on (with other glaucoma drug)", "Switch from prior treatment", "Initial monotherapy" and "Initial combination therapy" were 2224 (73.7\%), 27

The original article can be found online at https://doi. org/10.1007/s12325-018-0863-1.

H. Tanihara $(\bowtie)$

Kumamoto University Hospital, Kumamoto, Japan

e-mail: tanihara@pearl.ocn.ne.jp

T. Kakuda $\cdot$ T. Sano $\cdot$ T. Kanno $\cdot$ R. Imada

W. Shingaki · R. Gunji

Post Marketing Surveillance Department, Kowa

Company, Ltd., Tokyo, Japan
(0.9\%), 385 (12.8\%), $326(10.8 \%)$ and 54 (1.8\%), respectively. The mean number \pm SD of glaucoma medicines concomitantly used by patients was $2.3 \pm 1.0$.

\section{Efficacy}

Changes in IOP were also analysed according to the treatment initiation patterns (Fig. 5). IOP was significantly reduced in all groups: $-2.8 \pm 4.2 \mathrm{mmHg}$ in 313 eyes in the "Initial monotherapy" group, $-6.7 \pm 9.4 \mathrm{mmHg}$ in 42 eyes in the "Initial combination therapy" group, $-1.9 \pm 4.2 \mathrm{mmHg}$ in 362 eyes in the "Switch from prior treatment" group, $-2.5 \pm 3.8$ in 2100 eyes in the "Add-on (only)" group, and $-3.8 \pm 4.0 \mathrm{mmHg}$ in 22 eyes in the "Add-on (with other glaucoma drug" group. 


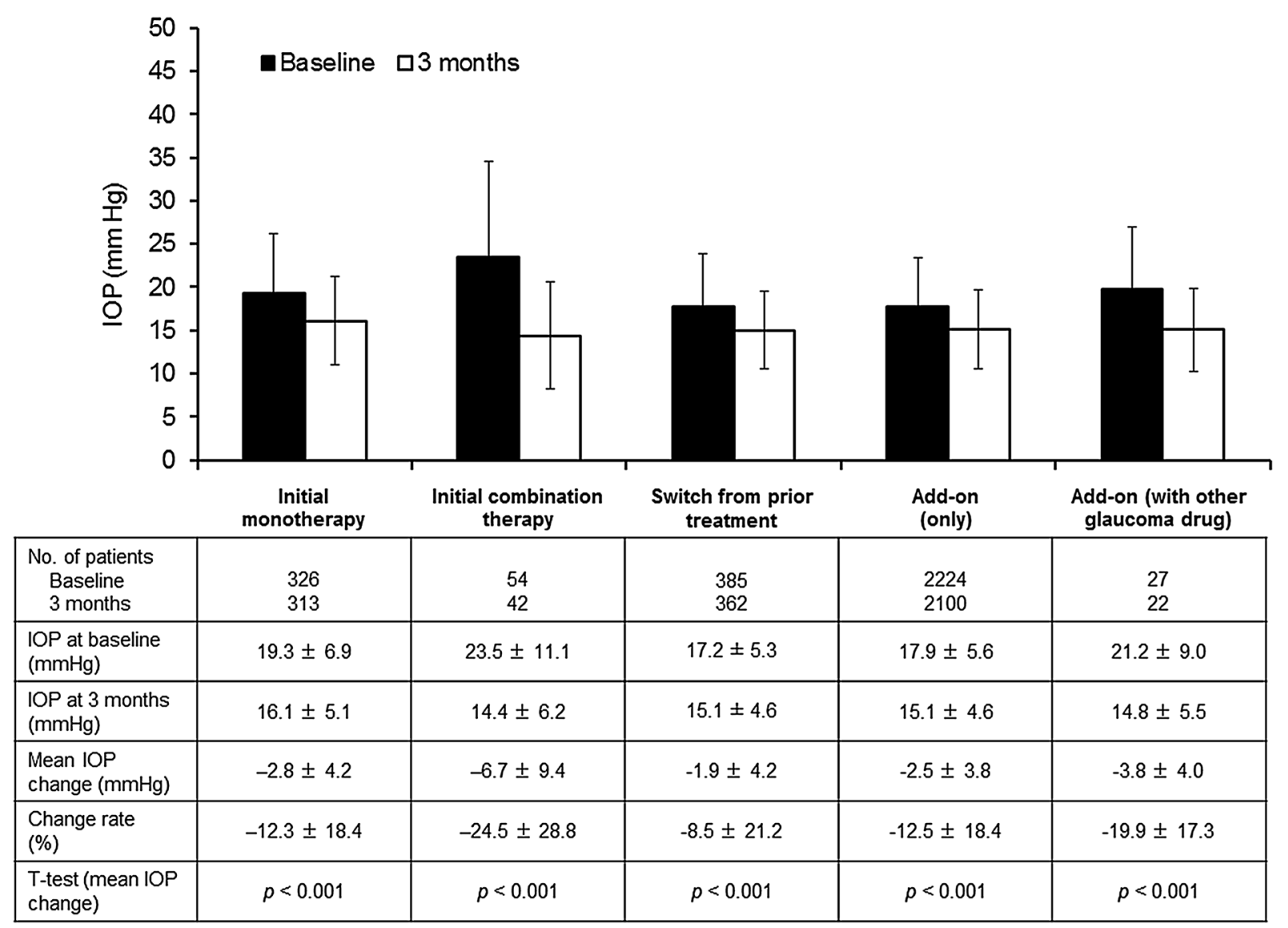

Fig. 5 Intraocular pressure changes in patients according to treatment initiation patterns. IOP intraocular pressure 